\title{
Enabling Solar Fuels Technology With High Throughput Experimentation
}

\author{
J. M. Gregoire ${ }^{1}$, J. A. Haber ${ }^{1}$, S. Mitrovic ${ }^{1}$, C. Xiang ${ }^{1}$, S. Suram ${ }^{1}$, P. F. Newhouse ${ }^{1}$, E. \\ Soedarmadji ${ }^{1}$, M. Marcin ${ }^{1}$, K. Kan ${ }^{1}$, D. Guevarra ${ }^{1}$, R. Jones ${ }^{1}$, N. Becerra ${ }^{1}$, E. W. Cornell ${ }^{2}$, J. Jin ${ }^{2}$ \\ ${ }^{1}$ Joint Center for Artificial Photosynthesis, California Institute of Technology, Pasadena, \\ California 91125, USA. \\ ${ }^{2}$ Engineering Division and Joint Center for Artificial Photosynthesis, Lawrence Berkeley \\ National Laboratory, Berkeley, California 94720, USA.
}

\begin{abstract}
The High Throughput Experimentation (HTE) project of the Joint Center for Artificial Photosynthesis (JCAP, http://solarfuelshub.org/) performs accelerated discovery of new earthabundant photoabsorbers and electrocatalysts. Through collaboration within the DOE solar fuels hub and with the broader research community, the new materials will be utilized in devices that efficiently convert solar energy, water and carbon dioxide into transportation fuels. JCAP-HTE builds high-throughput pipelines for the synthesis, screening and characterization of photoelectrochemical materials. In addition to a summary of these pipelines, we will describe several new screening instruments for high throughput (photo-)electrochemical measurements. These instruments are not only optimized for screening against solar fuels requirements, but also provide new tools for the broader combinatorial materials science community. We will also describe the high throughput discovery, follow-on verification, and device implementation of a new quaternary metal oxide catalyst. This rapid technology development from discovery to device implementation is a hallmark of the multi-faceted JCAP research effort.
\end{abstract}

\section{INTRODUCTION}

The widespread deployment of new energy technologies requires discovery and development of new functional materials. ${ }^{1}$ Artificial photosynthesis is a next-generation energy technology with several substantial materials challenges. ${ }^{2,3}$ Proposed designs for an artificial photosynthesis device or solar fuel generator involve coupling of electrocatalysts to light absorbing semiconductors to provide solar-driven photoelectrochemical reactions. ${ }^{2,3}{ }^{4}$ Successful development of such a device requires discovery of both photoabsorbers and electrocatalysts for the pertinent reactions. Given the limited solar irradiation power density, generation of fuel at market-relevant levels will require a large-scale, distributed technology., 6 As a result, desirable traits for new high performance materials include high earth abundance, facile synthesis methods and insensitivity to small variations in composition. To identify new photoabsorbers and electrocatalysts with these traits, we are building a high throughput pipeline for accelerated materials discovery.

The development of the accelerated discovery pipeline within a solar fuels research center provides very powerful capabilities with respect to the design and operation of the pipeline. As discussed below, the performance screening metrics employed in the pipeline are developed according to the specifications of the directed research and device prototype experts. Another important capability, which is not discussed further here, is the rapid incorporation of 
newly discovered materials into a solar fuels testbed. This capability shortens the time lapse between high throughput discovery and technology demonstration from years to weeks.

\section{EXPERIMENT}

The accelerated discovery pipeline contains 4 primary sectors as shown in Figure 1, three of which involve the development of new experimental equipment and techniques: Materials Synthesis, (Light-absorber and Electrocatalyst) Screening, and Characterization. The fourth sector is Data Informatics and Distribution and involves data connectivity to the other sectors and to users. To provide a synopsis of the pipeline experimental capabilities, we describe the materials synthesis via inkjet printing and the Light-absorber screening via photocurrent measurements.

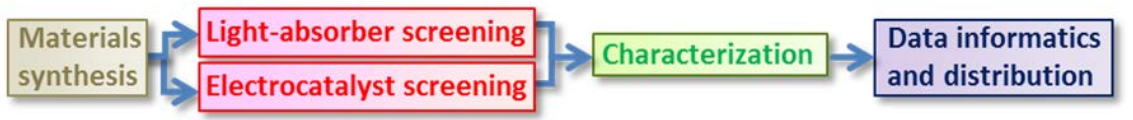

Figure 1: Sectors of the accelerated discovery pipeline, with the screening sector split for the 2 general material functions of light absorption and electrocatalysis.

\section{Printed discrete libraries}

Discrete composition libraries have been used extensively for the combinatorial screening of new composition spaces. The composition space of $n$ components contains $n-1$ dimensions. For creating a library of composition samples on a 2-D substrate, convenient patterns can be used for $n<4$ : a line for $n=2$ and a triangle for $n=3$. Larger values of $n$ require that the composition space be unfolded onto the 2-D surface. An example strategy for achieving this is shown in Figure 2 for quaternary ( $n=4$ ) composition space.

Another important parameter in the design of a discrete composition library is the concentration step size $\delta$. Table I shows the number of unique compositions for several values of $n$ and $\delta$, demonstrating the rapid increase in discrete library size with respect to increasing values of $n$ and decreasing values of $\delta$. For unexplored composition spaces, using a fine composition step is desirable to mitigate the possibility of missing a new high performance material. For the application of photoelectrochemistry, such as the example shown below, the sensitivity of performance to phase purity also motivates the use of small values of $\delta$. Figure 2 contains the $n=4, \delta=3.33 \%$ library layout synthesized using inkjet printing for the exploration of pseudoquaternary oxide composition spaces, such as $(\mathrm{Bi}-\mathrm{V}-\mathrm{Ni}-\mathrm{Fe}) \mathrm{O}_{\mathrm{x}}$.

Table I: The number of unique compositions in a discrete composition library is shown for several values of the number of components $n$ and composition steps $\delta$. The number of $\delta$ steps between $0 \%$ and $100 \%$ is also listed.

\begin{tabular}{|c|c|c|c|c|c|}
\hline num. steps & 10 & 20 & 30 & 40 & 50 \\
\hline$\delta$ & $10 \%$ & $5 \%$ & $3.33 \%$ & $2.5 \%$ & $2 \%$ \\
\hline$n=2$ & 11 & 21 & 31 & 41 & 51 \\
\hline$n=3$ & 66 & 231 & 496 & 861 & 1,326 \\
\hline$n=4$ & 286 & 1,771 & 5,456 & 12,341 & 23,426 \\
\hline$n=5$ & 1,001 & 10,626 & 46,376 & 135,751 & 316,251 \\
\hline$n=6$ & 3,003 & 53,130 & 324,632 & $1,221,759$ & $3,478,761$ \\
\hline
\end{tabular}


Inkjet printing of elemental precursors is a rapidly emerging technology in high throughput materials science. ${ }^{7-9}$ Commercially available printers offer an economical method for dispensing controlled quantities of “inks” containing elemental precursors. Different printer vendors offer various levels of speed, dispensing mechanism and chemical compatibility. The library discussed below was synthesized using a modified Epson Stylus Pro 4880. A recent report by Liu et $\mathrm{al}^{8}$ provides a strategy for producing elemental inks for this printing technology. This strategy uses ethanol as the primary solvent and dissolved block copolymers that can be used to control the solution viscosity.
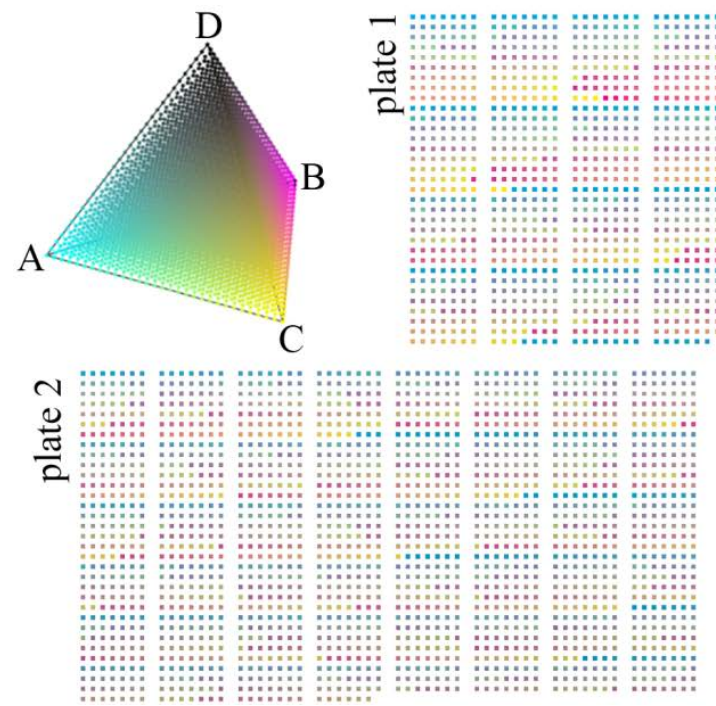
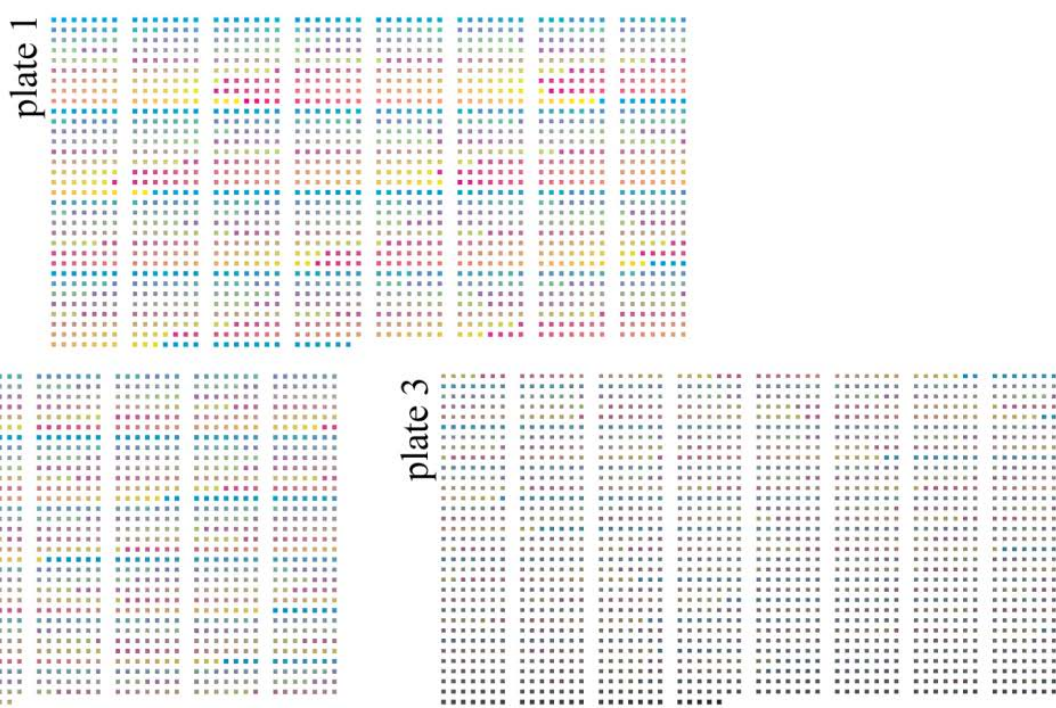

Figure 2: The discrete quaternary composition space with $\delta=3.3 \%$. The tetrahedron composition plot shows the 5456 compositions colored by the cyan-magenta-yellow-black coloring scheme. These compositions are laid out on a grid of $1 \mathrm{~mm}$ spots with $2 \mathrm{~mm}$ pitch such that three $4 \times 6$ ” plates contain the full composition space.

The Bi-V-Fe-Ni composition library discussed below was synthesized from four inks, each with an elemental precursor $\left(\mathrm{Bi}\left(\mathrm{NO}_{3}\right)_{3} \cdot 5 \mathrm{H}_{2} \mathrm{O}, \mathrm{VO}(\mathrm{i}-\mathrm{OPr})_{3}, \mathrm{FeCl}_{3}, \mathrm{Ni}\left(\mathrm{NO}_{3}\right)_{2} \cdot 6 \mathrm{H}_{2} \mathrm{O}\right.$ obtained from Sigma Aldrich). Using vigorous stirring, $5 \mathrm{mmol}$ of each metal precursor, $6 \mathrm{mmol}$ of $\mathrm{HNO}_{3}$ and $0.5 \mathrm{~g}$ of P123 ( $\mathrm{EO}_{20} \mathrm{PO}_{70} \mathrm{EO}_{20}, \mathrm{MW}=5800$; Sigma Aldrich) were dissolved into $45 \mathrm{~mL}$ ethanol. The inks were loaded into independently-operated channels of the printer and dispensed according to the discrete library design of Figure 2 with $1 \mathrm{~mm}^{2}$ printed on a $2 \mathrm{~mm}$ pitch. Each sample spot received an equal total volume of ink, providing approximately $10 \mathrm{nmol}$ of metal in each sample. This printing platform is a very fast deposition technique, with the 5456-member library requiring less than 20 minutes of printing time.

To provide electrical access to all samples, the library was deposited onto glass plates coated with $400 \mathrm{~nm}$ of fluorine-doped tin oxide (FTO) (TEC15 glass supplied by Hartford Glass Co., Hartford, IN; FTO sheet resistance 12-14 Ohms per square). The library of mixed metal precursor solutions was dried and calcined in air at $500{ }^{\circ} \mathrm{C}$ for 6 hours in a box furnace. This calcination efficiently volitalized and removed the precursor ligands and other ink components, and additionally yielded oxidation of the metals. The resulting samples were porous, noncontiguous metal oxide coatings with nominal thickness of approximately $300 \mathrm{~nm}$. Each sample was well adhered to the FTO coating and was contained within the designed $1 \mathrm{~mm}^{2}$ area. 


\section{Photocurrent screening}

To enable solar fuels technology, photo-absorbers must not only absorber light, but also transfer the photo-generated electrons or holes to solution via an electrochemical reaction. The photo-generated electrochemical current is thus used as a primary figure of merit (FOM) for these material libraries. Since the samples in the composition libraries share a common electrical contact, independent 3-electrode electrochemical measurements require sample-isolated solution contact. We recently developed a scanning droplet cell (SDC) optimized for performing electrochemical and photo-electrochemical measurements with unprecedented throughput. ${ }^{10}$ This apparatus was used to measure the photocurrent of the 5456 (Bi-V-Ni-Fe) $\mathrm{O}_{x}$ compositions.

For complex electrochemical reactions, such as the oxygen evolution reaction (OER) required for artificial photosynthesis, photocurrent is often kinetically limited by the catalytic activity of the electrode surface. Due to this kinetic limitation, photoabsorbers are generally coupled with active catalysts to create a photocatalytic electrode. While direct measurement of the OER photocatalytic activity can be performed using the SDC, we first want to map the photocurrent of new photoabsorber libraries without catalysis limitations. This limitation can be circumvented by incorporating a facile redox couple into solution.

The (Bi-V-Ni-Fe) $\mathrm{O}_{\mathrm{x}}$ library was screened in a solution composed of $50 \mathrm{mM}$ potassium ferrocyanide and $50 \mathrm{mM}$ potassium ferricyanide in water, where the solution potential was poised by the $\left[\mathrm{Fe}(\mathrm{CN})_{6}\right]^{4-} /\left[\mathrm{Fe}(\mathrm{CN})_{6}\right]^{3-}$ redox couple (360 $\mathrm{mV}$ vs. $\mathrm{NHE}$ ). The oxidized form, potassium ferrocyanide, served as an accepter of photo-generated holes in the n-type photoabsorbers. With solution contact to an individual composition sample serving as the working electrode, the electrochemical cell was completed using Pt wire reference and counter electrodes.

Short-circuit chronoamperometric measurements lasting $10 \mathrm{~s}$ were performed with illumination toggled at $2 \mathrm{~Hz}$ between no illumination and $2.5 \mathrm{~mW}$ from a $385 \mathrm{~nm}$ light emitting diode (LED). The incident power on the working electrode was measured before the high throughput experiment using a transparent quartz plate contacted by the droplet cell with a standard power meter pressed against the other side of the plate in transmission geometry. Each sample exhibited negligible dark current, as expected for the short circuit condition. The differential current under illumination was calculated for each sample, providing the desired measurement of photocurrent.

\section{DISCUSSION}

Example chronoamperometry data for 2 compositions is shown in Figure 3, which shows an initial transient current and a low-noise square wave commensurate with the illumination cycles. The measured photocurrent for all samples is shown in Figure 4. The spatial map of the photocurrent for all 3 plates gives the appearance that there are a handful of high photocurrent outliers. In Figure 4b, the data are folded back into the quaternary composition space with some transparency of the points allowing some visibility into the interior of the tetrahedron. For this library, no significant compositional trends exist in the interior of the tetrahedron, and the most pertinent composition trends are on the ternary faces of the tetrahedron as shown in Figure 4c. The $\mathrm{Bi}-\mathrm{V}$ binary line exhibits an expected compositional trend as $\mathrm{BiVO}_{4}$ is a well-known photoanode. The surprising composition trend is in the $\mathrm{Bi}-\mathrm{V}$-Fe space where a line of near equal photocurrent extends from $\mathrm{Bi}_{0.5} \mathrm{~V}_{0.5}$ to $\mathrm{Bi}_{0.13} \mathrm{~V}_{0.8} \mathrm{Fe}_{0.07}$. This discovery motivates further 
investigation of this composition region as the underlying materials science is not understood. It is worth noting that the plotted and quoted compositions are those intended from the library design. The printing passed basic quality control tests but the as-prepared compositions need to be measured as do the post-electrochemistry compositions, as the sample compositions may have been altered by chemical or electrochemical dissolution.

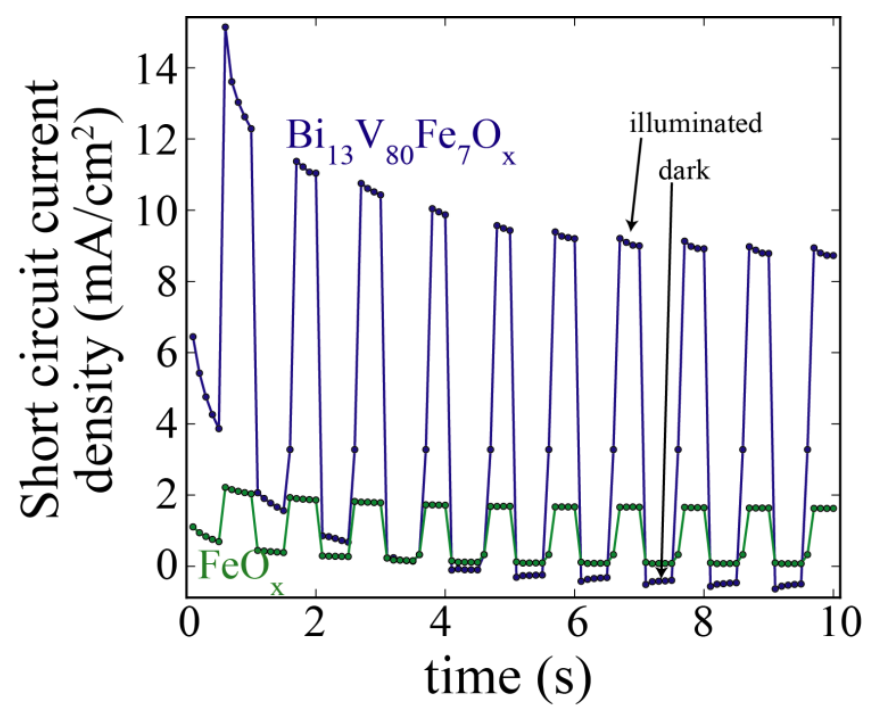

Figure 3: Chopped-illumination chronoamperometry data for 2 representative compositions. The figure of merit is the photocurrent, calculated as the difference in short circuit current between dark and illuminated portions of the illumination cycles.

It is worth noting that $\mathrm{FeO}_{\mathrm{x}}$ is a well-studied photoanode material and that this composition end point is a local maximum in the photocurrent map of Figures 4b-4c. As shown in Figure 3, this sample exhibits a photocurrent of $1.6 \mathrm{~mA} \mathrm{~cm}^{-2}$. In the false color maps, this photocurrent appears low due to the relatively large global maxima near $10 \mathrm{~mA} \mathrm{~cm}{ }^{-2}$. These photocurrents are well above the noise level of the measurement, as photocurrents of $10 \mu \mathrm{A} \mathrm{cm}$ have been detected by this technique.

The photocurrent map in Figure 4a demonstrates the high fidelity of the SDC measurement. The highly active compositions neighbor inactive compositions, and no cross-talk is evident in the measurements. Any spatial or temporal artifacts are negligible compared to the composition-dependent photocurrent. Figure 4c demonstrates the utility of the 3.3\% composition steps, as a library with $10 \%$ steps would have completed missed the compositional trend originating from the known $\mathrm{Bi}_{0.5} \mathrm{~V}_{0.5} \mathrm{O}_{\mathrm{x}}$ composition, and a library with $5 \%$ steps would have appeared more like a single point outlier on the $\left(\mathrm{Bi}_{1-\mathrm{y}} \mathrm{V}_{\mathrm{y}} \mathrm{Fe}_{0.05}\right)$ line rather than the wellestablished trend. 


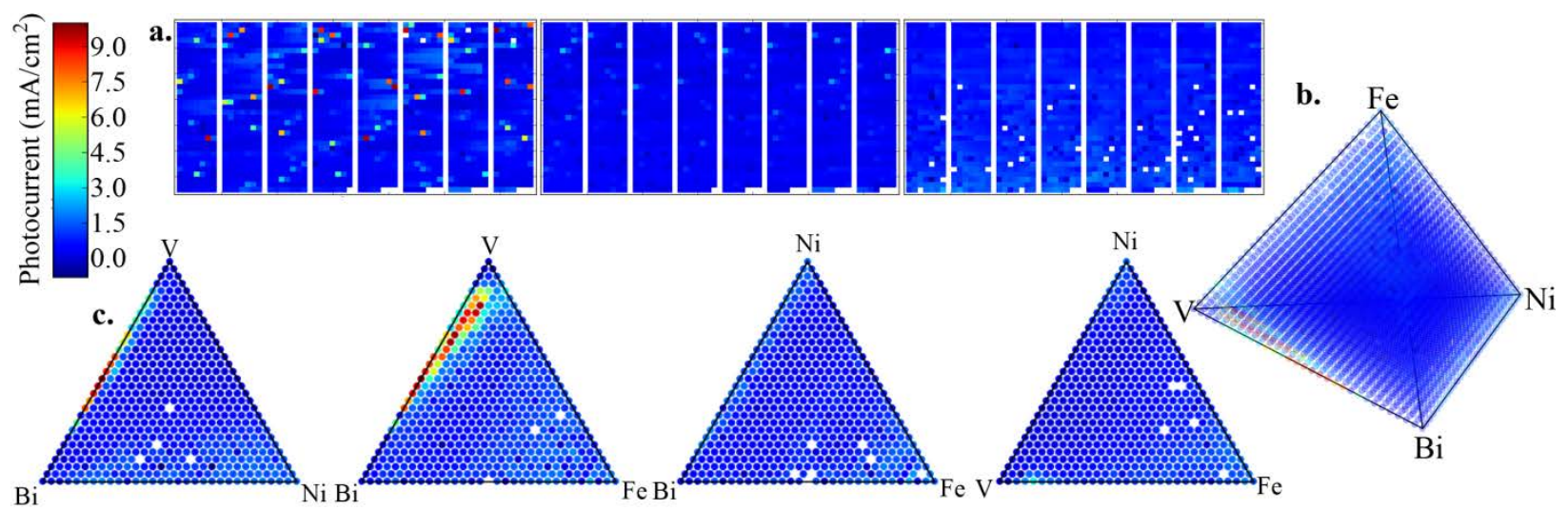

Figure 4: Using the common false color scale at the upper left, the photocurrent is mapped a. as a function of position on the 3 library plates, $\mathbf{b}$. on the tetrahedron representing the pseudo-quaternary composition space and $\mathbf{c}$. on the 4 pseudo-ternary faces of the tetrahedron. The missing data points correspond to samples for which a photocurrent is not reported due to poor quality chronoamperometry data.

\section{CONCLUSIONS}

While high throughput methods can be applied to a variety of technologies, considerable effort is required to develop techniques that screen material libraries for a particular function with a target application. For the primary functional materials in a solar fuels generator, electrocatalysts and light absorbers, we have developed high throughput screening tools that employ conditions and metrics directly applicable to the target technology. The screening tools are an integral part of a larger accelerated discovery pipeline, which is partially portrayed with the investigation of $(\mathrm{Bi}-\mathrm{V}-\mathrm{Ni}-\mathrm{Fe}) \mathrm{O}_{\mathrm{x}}$ light absorbers. The discovery of new photoabsorbers in this pseudo-quaternary space includes the design of a discrete composition grid, preparation and deposition of elemental precursor inks, and measurement of photo-generated electrochemical current. Surprising composition-performance trends are observed, motivating the movement of this materials library through the remainder of the discovery pipeline, specifically the characterization of the materials properties and the application of data analytics to reveal the underlying composition-property-performance relationships.

\section{ACKNOWLEDGMENTS}

This Material is based upon work performed by the Joint Center for Artificial Photosynthesis, a DOE Energy Innovation Hub, supported through the Office of Science of the U.S. Department of Energy (Award No. DE-SC0004993). The authors thank the entire JCAP team for assistance with the design and implementation of the accelerated discovery pipeline.

\section{REFERENCES}

1. Pinaud, B. A.; Benck, J. D.; Seitz, L. C.; Forman, A. J.; Chen, Z. B.; Deutsch, T. G.; James, B. D.; Baum, K. N.; Baum, G. N.; Ardo, S.; Wang, H. L.; Miller, E.; Jaramillo, T. F., Technical and economic feasibility of centralized facilities for solar hydrogen production via photocatalysis and photoelectrochemistry. Energy \& Environmental Science 2013, 6, (7), 19832002. 
2. Walter, M. G.; Warren, E. L.; McKone, J. R.; Boettcher, S. W.; Mi, Q. X.; Santori, E. A.; Lewis, N. S., Solar Water Splitting Cells. Chemical Reviews 2010, 110, (11), 6446-6473.

3. Bard, A. J.; Fox, M. A., Artificial Photosynthesis - Solar Splitting of Water to Hydrogen and Oxygen. Accounts of Chemical Research 1995, 28, (3), 141-145.

4. $\quad$ Haussener, S.; Xiang, C. X.; Spurgeon, J. M.; Ardo, S.; Lewis, N. S.; Weber, A. Z., Modeling, simulation, and design criteria for photoelectrochemical water-splitting systems. Energy \& Environmental Science 2012, 5, (12), 9922-9935.

5. Basic research needs for solar energy utilization; U.S. Department of Energy (DOE), Office of Basic Energy Sciences,: Washington, D.C., 2005.

6. Lewis, N. S., Toward cost-effective solar energy use. Science 2007, 315, (5813), 798801.

7. Calvert, P., Inkjet printing for materials and devices. Chemistry of Materials 2001, 13, (10), 3299-3305.

8. $\quad$ Liu, X. N.; Shen, Y.; Yang, R. T.; Zou, S. H.; Ji, X. L.; Shi, L.; Zhang, Y. C.; Liu, D. Y.; Xiao, L. P.; Zheng, X. M.; Li, S.; Fan, J.; Stucky, G. D., Inkjet Printing Assisted Synthesis of Multicomponent Mesoporous Metal Oxides for Ultrafast Catalyst Exploration. Nano Letters 2012, 12, (11), 5733-5739.

9. $\quad$ Gregoire, J. M.; Xiang, C.; Mitrovic, S.; Liu, X.; Marcin, M.; Cornell, E. W.; Fan, J.; Jin, J., Combined Catalysis and Optical Screening for High Throughput Discovery of Solar Fuels Catalysts. Journal of the Electrochemical Society 2013, 160, (4), F337-F342.

10. Gregoire, J. M.; Xiang, C. X.; Liu, X. N.; Marcin, M.; Jin, J., Scanning droplet cell for high throughput electrochemical and photoelectrochemical measurements. Review of Scientific Instruments 2013, 84, (2). 